\title{
Vivir el conflicto lejos de los campos de batalla. La comunidad alemana del Perú y la Primera Guerra Mundial
}

Vivre le conflit loin des champs de bataille. La communauté allemande du Pérou et la Première Guerre mondiale

Living the conflict far from the battlefields. The German community of Peru and the First World War

\section{Guillemette Martin}

\section{(2) OpenEdition}

\section{Journals}

Edición electrónica

URL: http://journals.openedition.org/bifea/7583

DOI: 10.4000/bifea.7583

ISSN: 2076-5827

Editor

Institut Français d'Études Andines

Edición impresa

Fecha de publicación: 1 agosto 2015

Paginación: 259-281

ISSN: 0303-7495

Referencia electrónica

Guillemette Martin, «Vivir el conflicto lejos de los campos de batalla. La comunidad alemana del Perú y la Primera Guerra Mundial », Bulletin de l'Institut français d'études andines [En línea], 44 (2) | 2015,

Publicado el 08 agosto 2015, consultado el 05 noviembre 2020. URL : http://journals.openedition.org/ bifea/7583 ; DOI : https://doi.org/10.4000/bifea.7583

\section{(c) (†) $\ominus$}

Les contenus du Bulletin de l'Institut français d'études andines sont mis à disposition selon les termes de la licence Creative Commons Attribution - Pas d'Utilisation Commerciale - Pas de Modification 4.0 International. 


\title{
Vivir el conflicto lejos de los campos de batalla. La comunidad alemana del Perú y la Primera Guerra Mundial ${ }^{*}$
}

\author{
Guillemette Martin ${ }^{* *}$
}

\begin{abstract}
Resumen
La conmemoración del Centenario de la Primera Guerra Mundial en 2014 representó la oportunidad de renovar los enfoques historiográficos tradicionales de la Gran Guerra, al analizar el conflicto desde nuevos espacios y nuevos cuestionamientos.

Desde esta perspectiva, el presente artículo estudia la movilización de la comunidad alemana del Perú a lo largo del conflicto a través de dos publicaciones germano-peruanas: La Guerra Gráfica y Germania. En un país claramente aliadófilo, el análisis de las representaciones elaboradas por la comunidad alemana del Perú nos invita a cuestionar las nociones de movilización y de cultura de la guerra, sobre un conflicto a la vez lejano y cercano para estos migrantes europeos.
\end{abstract}

Palabras clave: Primera Guerra Mundial, Perú, comunidad alemana, movilización, propaganda, prensa

* Parte de los resultados de la investigación presentados en el presente artículo fueron expuestos en el marco del «XXI ${ }^{e}$ Colloque Interdisciplinaire de l'AÉDDHUM», organizado en marzo de 2014 en la ciudad de Montreal. El texto de esta ponencia, redactada en francés y en una versión reducida, se encuentra en proceso de publicación en las ediciones Cambridge Scholars.

** Becaria posdoctoral del Programa de Becas Posdoctorales en la UNAM. E-mail: martinguillemette@ yahoo.fr 
Vivre le conflit loin des champs de bataille. La communauté

\section{allemande du Pérou et la Première Guerre mondiale}

\section{Résumé}

Commémoré en 2014, le Centenaire de la Première Guerre mondiale a représenté une opportunité sans précédent de renouvellement de l'approche historiographique de la Grande Guerre, en l'analysant sans précédent de renouvellement de l'approche historiographique de

Dans cette perspective, le présent article analyse la mobilisation de la communauté allemande du Pérou pendant le conflit, au travers de deux publications germano-péruviennes : La Guerra Gráfica et Germania.

Au sein d'un pays largement alliadophile, I'analyse des représentations mises en œuvre par la communauté allemande du Pérou pendant la Cuerre nous invite à réinterroger les notions de mobilisation et de

culture de la guerre, pendant un conflit à la fois proche et lointain pour l'Amérique latine.

Mots-clés : Première Guerre mondiale, Pérou, communauté allemande, mobilisation, propagande, presse

Living the conflict far from the battlefields. The German community of Peru and the First World War

\section{Abstract}

Commemorated in 2014, the Centenary of the First World War represented an opportunity to renew traditional historical studies of the Great War by developing an analysis of the conflict from new spaces and with new questions. From that perspective, this paper studies the mobilization of the German community of Peru during the First World War based on two German-Peruvian publications: La Guerra Gráfica and Germania.

In a country that was clearly pro-Allies, an analysis of the representations created by the German community of Peru is an invitation to interrogate the notions of mobilization and of the culture of war during a conflict that was both close and distant at the same time for these European migrants.

Key words: World War I, Peru, German community, mobilization, propaganda, press

\section{INTRODUCCIÓN}

La conmemoración del Centenario de la Primera Guerra Mundial (2014-2018) representa la oportunidad de renovar los enfoques historiográficos clásicos sobre la Gran Guerra, desde nuevos espacios y nuevas hipótesis. Desde esta perspectiva América Latina hoy día es objeto de un interés renovado por parte de los historiadores.

A través de diversos estudios se ha considerado el impacto multifacético del conflicto en países que se mantuvieron al margen de la contienda. En un artículo reciente, el historiador Olivier Compagnon propuso un panorama general de las consecuencias de la Primera Guerra Mundial en el espacio latinoamericano, al mismo tiempo que realizó un interesante inventario historiográfico, en el cual señala algunos campos de investigación novedosos que se están desarrollando en la actualidad (Compagnon, 2013b: 533-555).

Los trabajos publicados en la última década sobre América Latina y la Primera Guerra Mundial inauguraron un enfoque cultural y discursivo del acontecimiento, al analizar la recepción del conflicto por las distintas sociedades latinoamericanas 1 . En el marco de esta última tendencia historiográfica, uno de los ejes analíticos más dinámicos es el estudio de la movilización de las comunidades europeas radicadas en América Latina. Entre diversos trabajos cabe mencionar los estudios pioneros de la historiadora argentina María Inés Tato sobre los europeos de Buenos Aires (Tato, 2011), así como las diferentes interpretaciones de la movilización británica, francesa e italiana en Argentina y en Uruguay desarrolladas por Álvaro Cuenca (Cuenca, 2006), Hernán Otero (Otero, 2009) y Emilio Franzina (Franzina, 2000). Estas investigaciones resultan trascendentales en cuanto a la renovación de la historiografía de la Gran Guerra, principalmente por dos razones. Primero porque permiten ampliar el marco de análisis del conflicto al inscribirlo realmente en una perspectiva de historia global; y segundo, porque nos invitan a explorar nuevos espacios de propaganda y de elaboración de representaciones sobre el conflicto, a partir del estudio de las movilizaciones originadas lejos de los campos de batalla, entre poblaciones europeas emigradas, pero plenamente implicadas en el conflicto.

El presente artículo pretende brindar una aportación a este campo historiográfico recientemente desarrollado, al analizar la movilización de las comunidades alemanas del Perú en el conflicto. Específicamente, se trata de presentar y analizar dos publicaciones financiadas y editadas por los alemanes del Perú durante la Primera Guerra Mundial: La Guerra Gráfica, revista ilustrada publicada por la comunidad alemana de Lima entre noviembre de 1914 y marzo de 1915, y Germania, semanario publicado en la ciudad amazónica de lquitos desde el mes de diciembre de 1916

Al valorar un material archivístico hasta la fecha ignorado por los historiadores2, el presente artículo se inscribe dentro de un verdadero vacío historiográfico entre los estudios recientes sobre la Gran Guerra y América Latina. Los estudios que tratan del impacto de la Primera Guerra Mundial en el Perú son escasos, por lo tanto la visibilidad de los archivos peruanos sobre el tema queda extremadamente limitada3. Por otro lado, mientras que la «germanofilia» de parte de la opinión latinoamericana ha sido analizada en algunos trabajos, principalmente para

1 Es el enfoque adoptado por Olivier Compagnon en su último libro, sobre la recepción del prime conflicto mundial por las sociedades argentina y brasileña (Compagnon, 2013).

Hasta la fecha, no se han publicado trabajos sobre La Guerra Gráfica y Germania. Tampoco se han referenciado estas publicaciones fuera del catálogo manual de la Hemeroteca de la Biblioteca Nacional del Perú, donde este material archivístico se encuentra actualmente disponible.

Entre las últimas investigaciones realizadas sobre la recepción del primer conflicto mundial por la sociedad peruana y su impacto cultural en este país andino, cabe mencionar los trabajos de Gérard Borras (en prensa), quien analizó las referencias a la Gran Guerra en los cancioneros limeños de la época. 
México y Argentina ${ }^{4}$, los estudios sobre la movilización de las comunidades alemanas instaladas en América Latina siguen siendo escasos5.

En esta perspectiva, el presente artículo tiene principalmente tres objetivos. El primero es contribuir a la documentación de la recepción del primer conflicto mundial por parte de la opinión peruana. El segundo objetivo es dar cuenta de las voces de las comunidades alemanas del Perú, esto a través del análisis de un discurso difundido a contracorriente de la prensa nacional peruana, para entonces ampliamente aliadófila. Para terminar, el tercer objetivo del presente trabajo es dar a conocer un material de archivo hasta la fecha nunca estudiado por los historiadores del Perú contemporáneo.

A partir de estas tres metas, el presente estudio esbozará algunos elementos de reflexión sobre la historia cultural de la Gran Guerra, cuestionando el sentido y la manifestación de las movilizaciones de las comunidades europeas instaladas en América Latina, así como las nociones de cultura y de propaganda de guerra.

\section{EL PERÚ Y LA GRAN GUERRA: DE LA NEUTRALIDAD A LA \\ RUPTURA DIPLOMÁTICA}

Relativamente ignorado por los especialistas de la Primera Guerra Mundial, el Perú sigue siendo un país marginal en la historiografía latinoamericanista de la Gran Guerra. Si bien existen algunos estudios específicos sobre el impacto económico del conflicto en el país andino 6 , las consecuencias sociales y políticas de la Gran Guerra en el Perú siguen siendo un tema todavía muy poco investigado de manera específica. Encontramos algunas referencias al primer conflicto mundial en estudios más generales. Por ejemplo, en el libro Historia de la República, el historiador Jorge Basadre propone algunas páginas detalladas sobre los «efectos inmediatos de la Primera Guerra Mundial»(Basadre, 2005: 154-165), así como sobre «las consecuencias económicas de la Guerra Mundial entre 1915 y 1918» (Basadre, 2005: 228-255), las cuales brindan al lector una perspectiva general sobre las profundas transformaciones monetarias y comerciales que conoció el Perú en estos años. En el marco más específico de la Historia de las Relaciones Internacionales,

${ }^{4}$ Estos trabajos suelen poner de relieve el papel desempeñado por los servicios de propaganda germánicos. Ver por ejemplo el artículo de María Inés Tato sobre la propaganda alemana en Argentina (2014). Sobre México, ver el artículo de Ingrid Schulze Schneider (1993).

De manera lógica, la movilización de las comunidades alemanas de América Latina durante el conflicto ha sido documentada en los dos países que recibieron la mayor parte de la migración alemana en el continente, es decir Brasil y Argentina. Sobre Brasil, ver el libro de Frederick C. Luebke, 1987; sobre Argentina el historiador alemán Valentin Kramer está realizando actualmente una serie de investigaciones sobre la movilización humanitaria de las mujeres alemanas de Rosario. - Sobre el impacto económico de la Primera Guerra Mundial en el Perú, ver el artículo de Heraclio Bonilla (1979) así como el trabajo de Victor Madueño (1981). Recientemente hemos propuesto una serie de reflexiones sobre el impacto social y económico del conflicto a nivel regional, analizando la evolución del comercio de la lana en la región de Arequipa durante los años de la guerra (Martin, en prensa)
Fabián Novak Talavera publicó también dos trabajos fundamentales en cuanto a las relaciones diplomáticas del Perú con Francia (Novak Talavera, 2005) y con Alemania (Novak Talavera, 2004). Realizados a partir de un análisis detallado de los archivos del Ministerio de Relaciones Exteriores peruano, ambos libros dedican un capítulo a las relaciones del Perú con ambas potencias europeas durante el primer conflicto mundial. Al igual que en la obra de Basadre, estos dos estudios no toman en cuenta la movilización de las comunidades europeas del Perú durante la guerra, ni tampoco la de los intelectuales peruanos. Sobre estos puntos en particular, todo queda por escribir. Sin embargo, los trabajos de Fabián Novak Talavera siguen siendo de una gran utilidad para la comprensión de la postura diplomática del Perú respecto a los países beligerantes, la cual resulta ser un preámbulo necesario a cualquier análisis de la movilización peruana en estos años.

Hasta 1917, y al igual que los demás países latinoamericanos, el Perú mantuvo una neutralidad oficial respecto a una contienda considerada como «entre grandes naciones y por grandes intereses sobre cuestiones extra-continentales, que no afectaban el honor de los países de América ni sus intereses vitales» ${ }^{7}$. A inicios de febrero de 1917, la línea de conducta del gobierno peruano respecto a las potencias beligerantes mostró una importante ruptura, cuando un barco comercial peruano, el Lorton, fue hundido por el ejército alemán cerca de las costas españolas (Morales Erroch, 2006: 107-124). El Perú exigió reparación al imperio alemán, quien se negó a acceder a las demandas peruanas, con el argumento de que el Lorton transportaba lo que se consideraba para entonces como «contrabando de guerra»8. En el otoño de 1917 después de intensos debates en las asambleas y en la opinión nacional peruana y alentado por la entrada en guerra de los Estados Unidos contra las potencias centrales, el nuevo canciller peruano Francisco Tudela y Varela decidió a su vez la ruptura de las relaciones diplomáticas entre su país y el imperio alemán.

\section{LA OPINIÓN PERUANA Y LA GUERRA}

Si bien es difícil esbozar con precisión los contornos de lo que podríamos llamar la «opinión pública peruana», en un país que presentaba para entonces altas tasas de analfabetismo, se pueden rescatar algunas tendencias del debate público, principalmente capitalino a lo largo del conflicto.

La prensa nacional representada entre otros por los periódicos limeños El Comercio y La Prensa, se hizo eco de las indecisiones del gobierno peruano en cuanto a

Principios neutrales formulados por Enrique de la Riva-Agüero, responsable de las relaciones exteriores del Perú bajo la presidencia de José Pardo, de 1915 a 1919, y citados por Jorge Basadre, 2005: 217.

8 El detalle de las negociaciones entabladas entre el gobierno peruano y el imperio alemán está disponible en un reporte oficial de 43 páginas publicado por el Ministerio de Relaciones Exteriores peruano, y conservado en su version digital en la Universidad de Florida, Estados Unidos: http:// ufdc.ufl.edu/UF00023460/00001/1j. 
su postura diplomática frente a las potencias beligerantes. En los primeros años de la guerra, El Comercio publicaba de manera regular una sección titulada «la conflagración europea», en la cual reproducía, sin hacer comentarios, los diversos telegramas y artículos llegados de la prensa europea, informando cada semana a sus lectores de los últimos movimientos militares en los frentes de batalla, del avance de las negociaciones diplomáticas entre los diferentes beligerantes, de las importantes evoluciones técnicas acarreadas por el conflicto, etc. En esta sección y hasta 1917 predominó cierta neutralidad. El Comercio publicaba tanto noticias de París, donde se encontraba uno de sus corresponsales (El Comercio, 19 de agosto de 1915: 2-3), como desde el «lado alemán» de la guerra (EI Comercio, 27 de enero de 1917: 3).

En 1917, el incidente del Lorton tuvo como consecuencia cierta inflexión ideológica de la prensa limeña, que cuestionó para entonces lo conveniente de la neutralidad del Perú en el conflicto (El Comercio, 11 de febrero de 1917: 1).

Si bien la orientación aliadófila de la opinión pública peruana resulta a veces difícil de determinar en las páginas de la prensa nacional, esta toma de posición antialemanes quedó mucho más clara entre los intelectuales de todo el país. Cabe mencionar los artículos publicados en El Comercio por la escritora y periodista de origen alemán Dora Mayer, en los cuales atribuía la total responsabilidad del conflicto al militarismo germánico (El Comercio, 18 de julio de 1915). Un mes antes, el intelectual trujillano Antenor Orrego Espinoza culpaba también el imperio alemán, en las páginas del periódico La Reforma de Trujillo (La Reforma, 2 de junio de 1915: 29). El año siguiente, el intelectual arequipeño Modesto Málaga publicó a su vez un largo artículo en el periódico local La Federación en el cual denunciaba las pretensiones germánicas de «esclavizar a las naciones» (La Federación, 2 de mayo de 1916: 2). Si bien no se pueden generalizar estas opiniones antialemanes a toda la intelectualidad peruana, cuya participación al debate público sobre la guerra queda todavía por investigar, se observa una clara tendencia de la opinión a favor de los Aliados.

En la perspectiva de contraponer esta orientación ampliamente aliadófila de la opinión, las comunidades alemanas de Lima e Iquitos decidieron crear sus propias publicaciones, únicos medios de difusión de un discurso diferente sobre la guerra.

\section{LOS EUROPEOS DEL PERÚ Y LA GUERRA}

Existen algunos trabajos dedicados a la historia de las diferentes comunidades europeas en el Perú10, sin embargo, no hemos podido encontrar un análisis

9 Titulado «La egolatría alemana», este artículo de Antenor Orrego Espinoza es citado por Germán Peralta Rivera (2011: 291-292). Antenor Orrego Espinoza es uno de los fundadores de la Bohemia de Trujillo y del movimiento intelectual «Grupo Norte» en los años 1920, en el cual participan importantes autores como César Vallejo y Victor Raúl Haya de la Torre.

10 Sobre la historia de los franceses del Perú, ver los trabajos de Pascal Riviale (2008). Sobre la historia de los alemanes en el Perú, ver el libro publicado por Diana Millies (2007), así como el libro de sistemático de la movilización de estas poblaciones migrantes durante la Gran Guerra.

No obstante, los archivos peruanos, empezando con la prensa limeña, muestran una importante movilización de los europeos de la capital peruana a lo largo del conflicto, al mismo tiempo que revelan fuentes documentales suficientes para llevar a cabo un primer estudio exploratorio de estas movilizaciones. Por ejemplo, el periódico El Comercio publicaba constantemente diversos avisos emitidos por la Cruz Roja británica y la Cruz Roja alemana con el fin de organizar actividades caritativas a favor de las victimas de guerra y de los soldados enviados al frente. La fundación del periódico L'Alliance, a inicios del conflicto y gracias al apoyo económico de la comunidad francesa de Lima, también mostró una manifestación clara de esta movilización11.

A inicios del siglo XX, la comunidad alemana del Perú seguía contando con muy pocos miembros. En un estudio de las migraciones alemanas a América, la historiadora Ingrid Schulze Schneider (Schulze Schneider, 1995: 261) menciona la cifra de 1100 alemanes para el año 1906 en el Perú. Si bien es cierto que los flujos migratorios entre Hamburgo y el Callao disminuyeron durante la Guerra, la comunidad alemana de Lima ganó alrededor de 250 nuevos integrantes, con la internación de ocho buques alemanes por el gobierno peruano en el puerto del Callao (Millies, 2007: 88)

Aunque poco numerosos, los miembros de la pequeña colonia alemana instalada en la capital participaron de manera activa en la vida cultural local. En los años 1910 crearon un colegio alemán (Deutsche Schule) con el fin de recibir a los alumnos alemanes de la ciudad de Lima. Iniciativa liderada por el comerciante alemán Fernando Oechsle, el colegio alemán pronto abrió sus puertas a los alumnos peruanos también y formó a reconocidos intelectuales peruanos, como por ejemplo Jorge Basadre.

Desde los primeros meses de la guerra, esta activa colonia alemana se organizó, al principio para ayudar a sus compatriotas en el frente, más tarde para proteger sus intereses económicos frente al establecimiento de «listas negras» por el gobierno peruano, en un contexto de creciente germanofobia (Millies, 2007: 87).

Es en este clima de hostilidad contundente que las comunidades alemanas del país tuvieron que defender su propia perspectiva sobre el conflicto. La creación de las dos publicaciones que nos interesan, La Guerra Gráfica, fundada en Lima en 1914 y Germania publicada en Iquitos a partir de 1916 representan una tentativa de respuesta a esta doble necesidad: por un lado difundir un discurso diferente sobre la guerra, y por el otro defender los intereses económicos y políticos de los alemanes instalados en el Perú.

Guillermo Godbersen (2002). Este último libro no es un trabajo científico en sí, sino más bien un libro-homenaje a la comunidad alemana del Perú.

Publicado desde inicios del año 1915 y hasta el final de la guerra, L'Alliance está distribuido por la empresa Nestlé en Lima. 


\section{GERMANIA, LA GUERRA GRÁFICA Y EL «LADO ALEMÁN» DE LA GUERRA}

El primer número de La Guerra Gráfica se publicó el 28 de noviembre de 1914 en Lima12, con el objetivo de brindar a sus lectores una información visual y gráfica del conflicto. La revista se imprimía en la imprenta del alemán Hermann Seer ubicada al número 365 de la Calle San Pedro. El primer artículo publicado por la Guerra Gráfica estaba dedicado a una presentación de los principales objetivos de la revista. En estas primeras líneas, el apoyo a Alemania no estaba claramente formulado. Se enfatizaba la voluntad de informar a los lectores de manera «imparcial», con el fin de equilibrar el debate apasionado que se daba sobre la guerra en el Perú (La Guerra Gráfica, 28 de noviembre de 1914: 2). Sin embargo, desde el segundo artículo aparecieron de manera mucho más contundente las orientaciones ideológicas del periódico:

No es nuestro fin al presentarnos ante el público neutral para quien escribimos, ni designar a nuestros infinitos adversarios, queremos, sí, que se nos oiga y después se juzgue lo que decimos con imparcialidad que debe ser la balanza de todo neutral.

Una inescrupulosa Política de Interés personal, envidia y venganza, ha unido en fraternal abrazo a tres naciones, que durante siglos se desconfiaron y se hicieron la guerra, donde y cuando se les presentaba la ocasión.

Esta unión nació con el único objeto,

de rebajar y destruir a un pueblo, que en competencia mundial y pacífica trabajaba con entusiasmo inquebrantable, en el engrandecimiento de la industria, del Comercio, de las ciencias y de todo lo que significaba el adelanto y bienestar de todo sin excepción [...]. Esta guerra sangrienta no la lleva Alemania para aumentar el brillo de la corona imperial, no y mil veces no! Esta lucha es la de un pueblo, que pelea por su honor, por su libertad y

${ }^{12}$ Conservado desde su primer número en la Hemeroteca de la Biblioteca Nacional del Perú, La Guerra Gráfica se publicaba semanalmente. El último número conservado es el número 18, publicado el 27 de marzo de 1915. por su existencia, y que solo por último estremo [sic.] ha tenido que tomar la espada en la mano [...] (La Guerra Gráfica, 28 de noviembre de 1914: 2) Firmado por «E. Polack Schneider», este artículo planteó la orientación ideológica de la publicación para los números siguientes, es decir: a contracorriente de las ideas ampliamente difundidas en la opinión peruana, Alemania no tenía porque asumir la responsabilidad —y la culpabilidad — del conflicto. Su lucha era legítima, frente a una alianza considerada como antinatural entre potencias históricamente enemigas (ahí podemos leer una referencia indirecta a la compleja relación franco-británica).

Si bien este artículo informa de las intenciones del periódico, el hecho de haber sido financiado por empresas alemanas instaladas en Lima no deja ninguna duda sobre las orientaciones ideológicas de la publicación. Las últimas páginas de cada número de La Guerra Gráfica reproducían una serie de avisos sobre los negocios alemanes de la capital peruana. Entre estas empresas figuraban la de Albert Hermann, comerciante de máquinas industriales, Ernesto Messner, dueño del Hotel Gran Cardinal, así como la Deutsche Wurstfabrik de Augusto Siegmund. Aunque no nos es posible determinar la amplitud de la difusión y del lectorado de La Guerra Gráfica por falta de una documentación complementaria, este poderoso patrocinio puede explicar la gran calidad material de la publicación, editada sobre un papel grueso y con una excelente calidad de impresión.

Fundado el 23 de diciembre de 1916 por la comunidad alemana de Iquitos ${ }^{13}$ el semanario Germania presenta un formato mucho más sencillo que La Guerra Gráfica, con una sola hoja impresa de ambos lados.

Desde un principio, sus objetivos fueron muy claros:

Un grupo de miembros de la colonia alemana, residente en esta ciudad y algunos de los numerosos amigos con que cuenta aquí la causa de los Imperios Centrales en la Guerra Europea, han resuelto dar a la publicidad, con el nombre de "GERMANIA", una publicación semanal, del formato de la presente, y que, sin herir la suceptibilidad [sic.], el patriotismo ni atacar la moral de los miembros de los países contra quien Alemania y sus aliados luchan, sirva para hacer conocer en esta región el verdadero estado de las operaciones bélicas, levantar las calumniosas informaciones que sobre esos países se han hecho recaer, dar una idea de su organización, finanzas, potencialidad económica, espíritu público, abnegación patriótica de sus hijos y grado de cultura alcanzado en todas las manifestaciones de su existencia como nación.

Se excluye de nuestra propaganda el insulto, el ridículo y la provocación a quienes no participen de nuestras ideas, limitándonos a una información verídica, serena, desapasionada y correcta de cuánto pueda ser interesante para nuestros intereses y favorable a la lucha que actualmente se libra por

${ }^{13}$ Cermania se imprimió en la Imprenta «El Oriente» de Iquitos. Los diferentes números de esta publicación están conservados hasta el 6 de octubre de 1917 en la Biblioteca Nacional del Perú, sumando un total de 42 números conservados. 

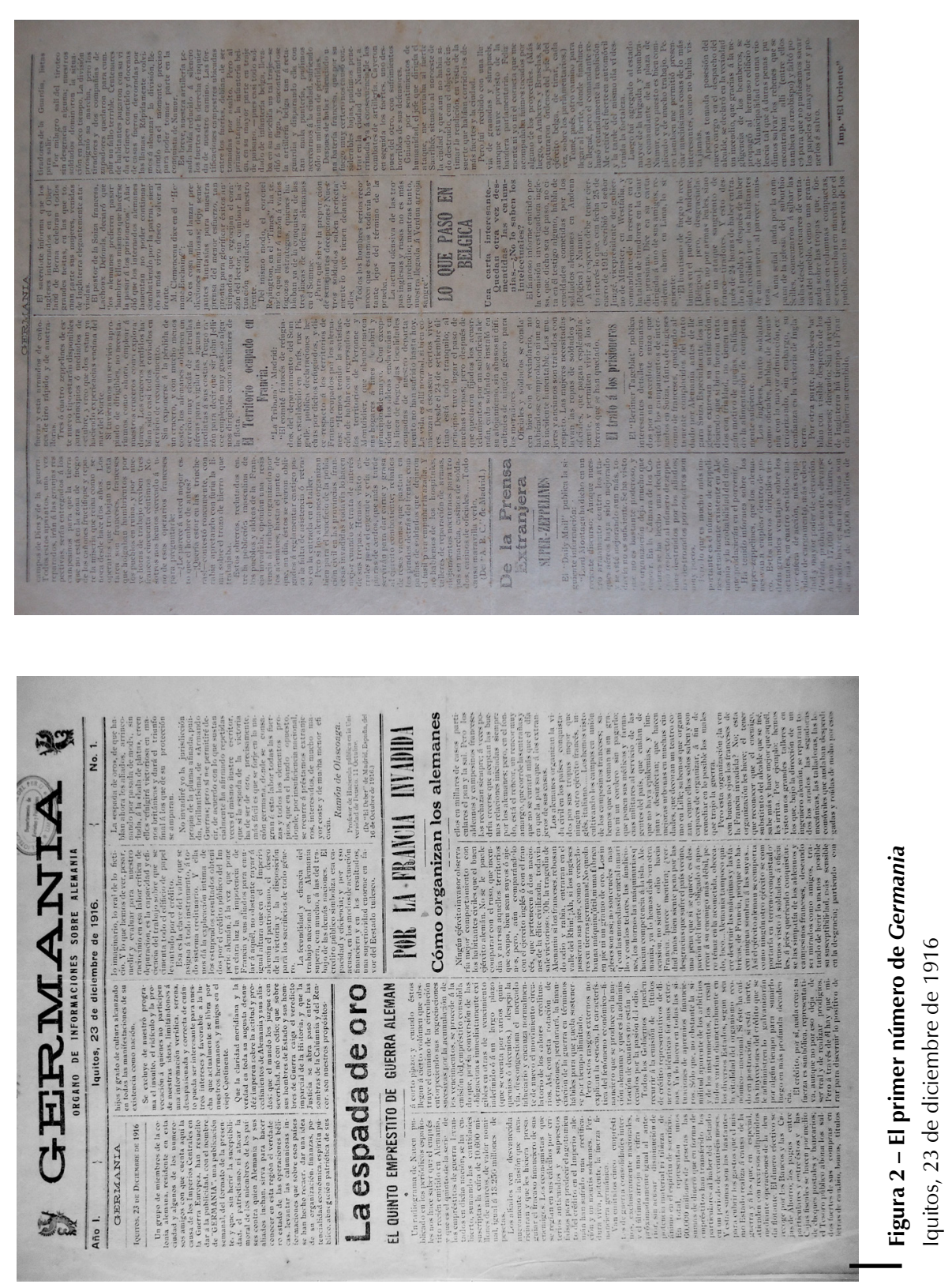

nuestros hermanos y amigos en el viejo Continente [...] (Germania, 23 de diciembre de 1916: 1)

Presentándose a sí mismo como un «órgano de informaciones sobre Alemania», el periódico Germania dedicaba la casi totalidad de sus páginas a la reproducción de artículos de opinión favorables a los Imperios Centrales, publicados en la prensa europea y alemana principalmente. Entre los periódicos regularmente mencionados figuraban publicaciones madrileñas como El Deber, A.B.C., El Correo Español, así como los periódicos alemanes Berliner Tageblatt o Hamburger Fremdeublatt. Germania también reproducía a menudo artículos publicados en los periódicos limeños, tales como El Comercio, El Perú y La Prensa. Limitándose muchas veces en proponer a sus lectores una revista de prensa sobre la guerra, Germania publicó muy pocos artículos propios. Sin embargo, este documento no deja de ser interesante para el historiador en la medida en que nos informa sobre el acceso de los lectores de provincia a una información internacional sobre el conflicto, así como sobre la difusión de un discurso germanófilo en la ciudad de lquitos.

La Guerra Gráfica, aunque proponía numerosos artículos de opinión original, también imprimió artículos publicados en la prensa extranjera. Reproducía por ejemplo, traducciones de la Illustrirte Geschichte des Welt Kriegs, del Berliner Tageblatt o del Neueste Nashrichte de Kiel.

En su noveno número, publicado el 23 de enero de 1915, La Guerra Gráfica ofreció a sus lectores una nueva sección, titulada «El Teatro de la Guerra», en la cual compartía las informaciones llegadas por cable. El periódico avisó a sus lectores de la parcialidad de esta información, difundida por las agencias de comunicación británicas:

La Guerra Gráfica, en su deseo de tener al corriente a sus lectores sobre los acontecimientos realizados en el teatro de la guerra dará semanalmente un resumen de los principales sucesos. Pero debemos llamar la atención, que las informaciones recibidas por el cable son vistas por el prisma inglés, por dominar esta nación todos los cables, con excepción de los pocos telegramas trasmitidos en forma lacónica de Alemania de la Estación Inalámbrica de Sayville, cerca de Nueva York y los cables provenientes de Chile de la Agencia Osterreich, información que debemos a la gentileza de un grupo de caballeros de la colonia alemana (La Guerra Gráfica, 23 de enero de 1915: 1).

En efecto, el acceso de la prensa germanófila a una información transmitida directamente por el imperio alemán parecía ser difícil en el Perú. A inicios del siglo XX el gobierno alemán se empeñó en construir su propia red radiotelegráfica, con el fin de independizarse del sistema telegráfico internacional británico. La compañía Telefunken había sido creada en 1903 con este fin. Como lo explica La Guerra Gráfica, a inicios de la guerra Telefunken logró enviar mensajes de larga distancia a su estación en los Estados Unidos, al mismo tiempo que entablaba negociaciones con el gobierno peruano para establecer en el país andino su propia central radiotelegráfica (Novak Talavera, 2004: 79-80). A pesar de estos esfuerzos, los servicios de comunicación alemanes tuvieron un impacto claramente menor al 
de los servicios británicos, quienes conservaban el monopolio de la información sobre la Guerra en el Perú14.

En esta perspectiva, la meta perseguida por La Guerra Gráfica y Germania de informar a los lectores peruanos sobre el lado alemán de la guerra, resultaba todavía más desafiante. Ambas publicaciones tenían entonces como tarea matizar, balancear y criticar las informaciones llegadas de Europa desde los despachos aliados, a la vez que publicar artículos favorables a Alemania, todo esto con el objetivo de ofrecer un contraste constante a las informaciones aliadófilas.

\section{MOVILIZACIÓN Y PROPAGANDA}

Para librar esta difícil lucha en contra de la propaganda aliada, Germania y La Guerra Gráfica recurrieron a una primera arma discursiva, la misma en ambas publicaciones: a través de una multiplicidad de ilustraciones, trataron de demostrar que Alemania era una potencia culta, generosa y civilizadora, cuya postura en la contienda resultaba ser, por ende, legítima. El argumento fungió como necesario contrapunto al argumento central de la propaganda aliada, que consistía en describir al pueblo alemán como bélico y violento15.

Desde su primer número y en esta perspectiva, La Guerra Gráfica reprodujo la contribución del periodista español Salvador Canals, en la cual se cuestionaba esta idea de la «barbarie alemana» tal como fue difundida por los Aliados:

Confieso que no me persuado a creer en la barbarie germánica ni en la exactitud de los hechos que se imputan a las tropas del Kaiser [...]. La característica de Alemania, de la Alemania que nos han pintado antes de la guerra, incluso sus enemigos en ésta, no es el ejército formidable, ni la industria prepotente, ni el comercio invasor, sino la cultura y su primera consecuencia: la disciplina social. La cultura alemana ha irradiado por el mundo entero, no habiendo rama alguna del saber donde no hayan dejado la huella, cuando menos, de sus métodos insuperables. Mucho antes de la guerra, mucho antes de haber en Inglaterra una opinión anti-alemana, los primeros pensadores ingleses atribuían la superioridad que Alemania iba alcanzando sobre su patria al crecimiento incesante de una cultura que en la Gran Bretaña parecía como estancada y en decadencia. Todo eso que se ha sintetizado en la bandera odiada del Made in Germany, verdadero pendón de guerra para el Reino Unido en la presente contienda, era, a juicio de los propios ingleses, el fruto de una enseñanza técnica en enormes

${ }^{14}$ Existen estudios detallados sobre la labor propagandística del gobierno alemán en América Latina durante la Primera Guerra Mundial, principalmente para México. Ver por ejemplo los trabajos de Ingrid Schulze Schneider (1993) y de Friedrich Katz (1981)

${ }^{15}$ Sobre esta oposición discursiva entre civilización y barbarie tal como dibuja el enfrentamiento ideológico y propagandístico entre aliadófilos y germanófilos en la prensa latinoamericana, ver las páginas 79 a 90 — «Civilisation française contre barbarie allemande» - del libro de Olivier Compagnon (2013a) y constantes progresos, en contraste con una cultura atacada de parálisis [...] (La Guerra Gráfica, 28 de noviembre de 1914: 6).

El autor de estas líneas plasmó un doble argumento, recurrente en ambas publicaciones: por un lado la referencia a la famosa «Kultur» alemana, admirada hasta por sus enemigos en el pasado; por el otro la decadencia cultural y moral de las potencias aliadas, argumento sobre el cual nos detendremos más adelante. Esta referencia a la «Kultur» alemana como conjunto de valores estéticos, políticos y morales que definen para entonces la identidad cultural alemana, se esbozó a menudo en las páginas de la Guerra Gráfica, con el fin de contrarrestar la representación de una Alemania «bárbara», elaborada por las potencias aliadas.

Se ha asegurado de Kansas a Kesan, de Kragujewaatsch a Ciudad del Cabo que los alemanes son 'Bárbaros'. No se debería decir que son algo justamente contrario que Bárbaros? [...] Todo lo bueno no existe en Alemania, pero hay una buena porción de antibarbarie en el Konigsberg de

Kant, en el Bonn de Beethoven, en el Berlin de Heimholtz y en el Nurnberg de Duren [...]. (La Guerra Gráfica, 26 de diciembre de 1914: 1).

Retomadas del periódico sueco Stockolms Dagblad, estas líneas ilustraban el argumento central de la Guerra Gráfica, publicación enteramente dedicada a una revaloración de la cultura e identidad alemanas.

Las cualidades militares y humanas demostradas por el ejército alemán durante el conflicto, también fueron subrayadas a menudo. La Guerra Gráfica mencionaba tanto el «admirable espionaje alemán» (La Guerra Gráfica, 5 de diciembre de 1914: 7), como el «triunfo de la artillería alemana» (La Guerra Gráfica, 19 de diciembre de 1914: 9). En su primer número, Germania también elaboró un verdadero elogio del ejército alemán, subrayando de manera algo contradictoria el gran respeto con el cual sus soldados serían recibidos en los pueblos franceses invadidos:

Ningún ejército invasor observaría mejor comportamiento con los habitantes civiles que el buen ejército alemán. [...] Hemos visto a soldados, a oficiales, a jefes queriendo conquistarse las simpatías de los aldeanos y campesinos franceses, deseando ser mirados como amigos, tratando de herir lo menos posible su susceptibilidad, más sensible en la desgracia; partiendo con ellos en millares de casos particulares el pan y la sal. [...] Los alemanes organizan la vida civil en los países conquistados por sus tropas mejor que ningún otro ejército francés, inglés, italiano... Los hemos visto cultivando los campos en unión de los campesinos franceses; sabemos que no toman ni un grano ni un cordero sin pagarlo; que ponen sus médicos y farmacias militares a servicio de las gentes del país, que cuidan de que no haya epidemias, y limpian y desinfectan; que hacen mejores urbanas en muchas ciudades [...]. (Germania, 23 de diciembre de 1916: 1).

Las ilustraciones publicadas por La Guerra Gráfica también alimentan esta imagen de un ejército alemán generoso y solidario, a través de elocuentes fotografías: 

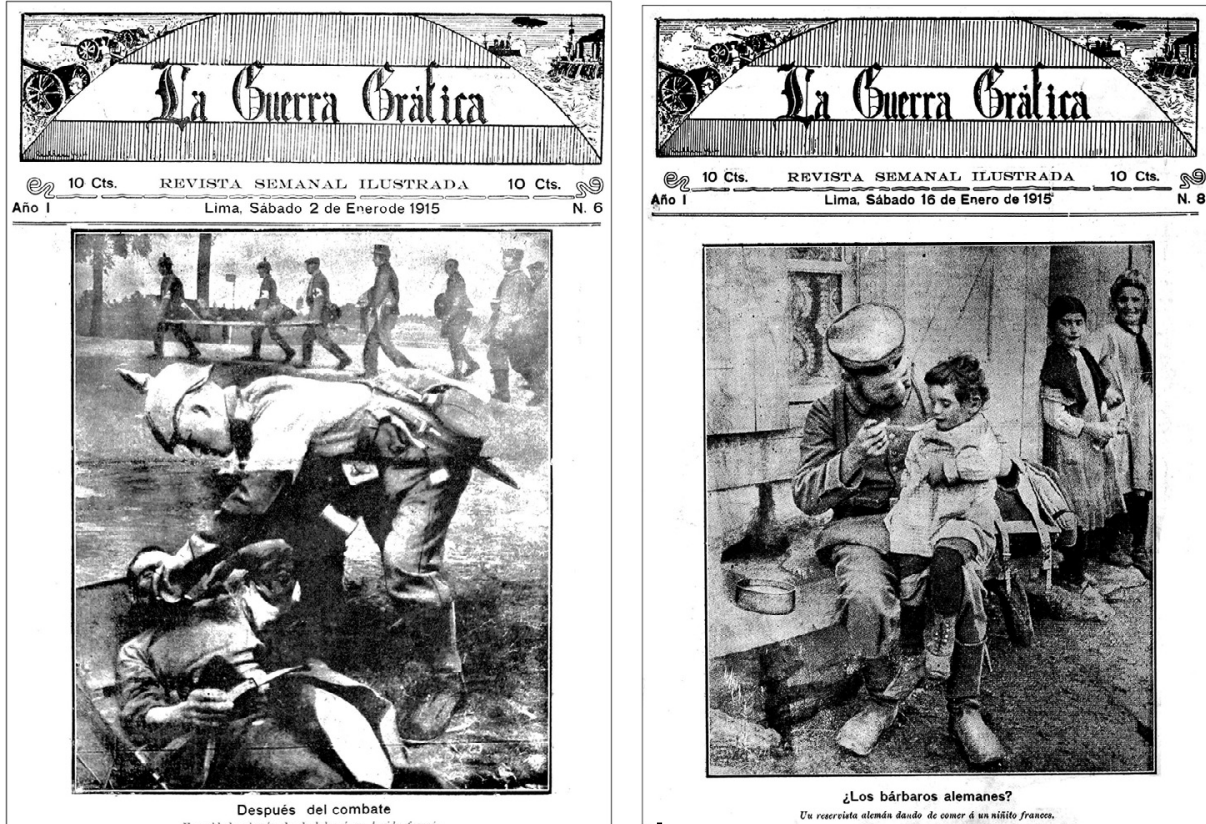

Figura 3 - «Después del combate. Un soldado alemán da de beber a un herido soldado a
francés»

Portada de La Guerra Gráfica, 2 de enero de 191

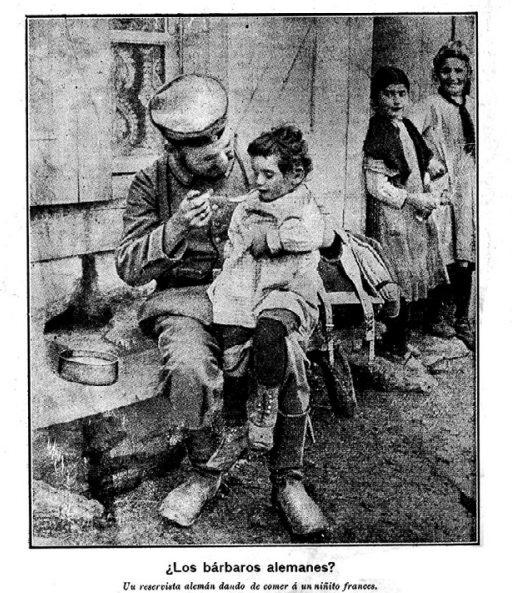

Figura 4 - «iLLos Bárbaros alemanes? Un reservista alemán dando de comer a un niñito francés»

Portada de La Guerra Gráfica, 16 de enero de 1915

En el mismo sentido, en el número 18 se reprodujo la fotografía de un soldado alemán sentado en medio de un grupo de niños sonriendo y comiendo, con este comentario:

El soldado alemán reparte su pan con el pueblo enemigo. Los niños de una aldea francesa conquistada, saborean gustosamente el pan alemán ( $L a$ Guerra Gráfica, 27 de marzo de 1915: 4).

Ahí el contrapunto es importante, porque en los primeros meses de la guerra, las tropas alemanas invadieron Bélgica con una gran brutalidad. Para entonces la prensa aliadófila articulaba gran parte de su propaganda alrededor de la denuncia de esta violenta invasión, como prueba de la barbarie germánica. En este contexto, uno de los principales objetivos de la propaganda alemana fue brindar otra imagen de su ejército.

Tanto La Guerra Gráfica como Germania pretendían mostrar que la bondad del ejército germánico no era más que el reflejo de la generosidad de la sociedad alemana en su conjunto, movilizada y solidaria. Ambas revistas publicaron diversos artículos sobre la Cruz Roja alemana, «la más importante por el número de sus ramificaciones y el inestimable valor de sus servicios», entre las diversas organizaciones vinculadas a la Cruz Roja internacional (Germania, 20 de enero de
1917: 1). En estos artículos se hablaba de las «cocinas económicas», destinadas a alimentar la población alemana en estos años de grave crisis, y

no porque Alemania esté en un estado de miseria, porque no hay ni rastro de ella, sino para probar que a pesar de todas las publicaciones hechas por los aliados, nunca conseguirán hacer morir de hambre a las mujeres y niños, ni a los hombres en Alemania (Germania, 20 de enero de 1917: 2). Contra la «barbarie alemana» muchas veces evocada por la propaganda aliada, Germania y La Guerra Gráfica quisieron mostrar una visión positiva de Alemania, de su cultura y de su ejército en una defensa que tiene como contrapunto lógico una acusación sistemática en contra de las potencias aliadas. Ambas publicaciones orientaban las más graves de sus acusaciones hacia Inglaterra, descrita como una nación cruel, violenta y deshonesta.

Sobre este último punto, cabe señalar una diferencia notable entre el discurso sobre Francia y el discurso sobre Inglaterra. Francia se encuentra humillada y desacreditada como potencia militar en La Guerra Gráfica, a través de caricaturas explícitas.

Realizadas especialmente para La Guerra Gráfica en los primeros meses del conflicto, estas caricaturas hablan por sí mismas: el ejército alemán conocía para entonces avances significativos entre las líneas defensivas francesas, autorizando cierto optimismo por parte de los Imperios Centrales. La segunda caricatura (fig. 6 ) es una representación de la «semeuse» (mujer sembradora), símbolo de la moneda francesa en esta época. Podemos ver ahí un juego de palabras con el «sol», la moneda peruana, que remite aquí al nuevo orden impuesto por el imperio alemán.

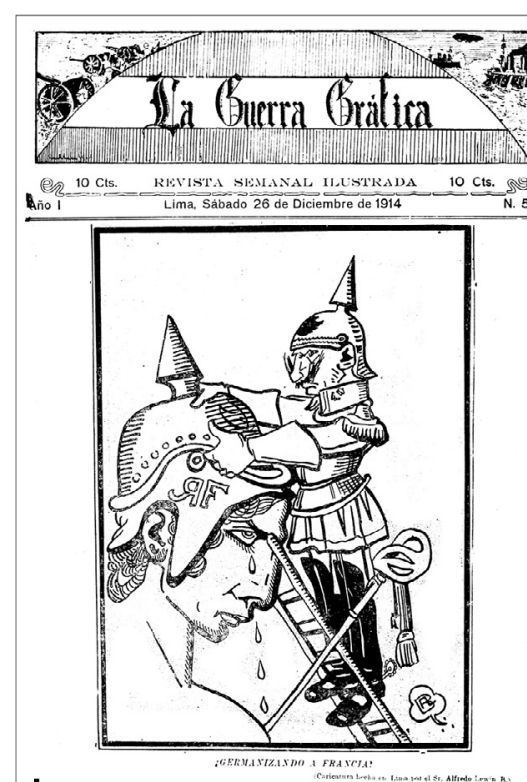

Figura 5 - «Germanizando a Francia !» La Guerra Gráfica, 26 de diciembre de 191
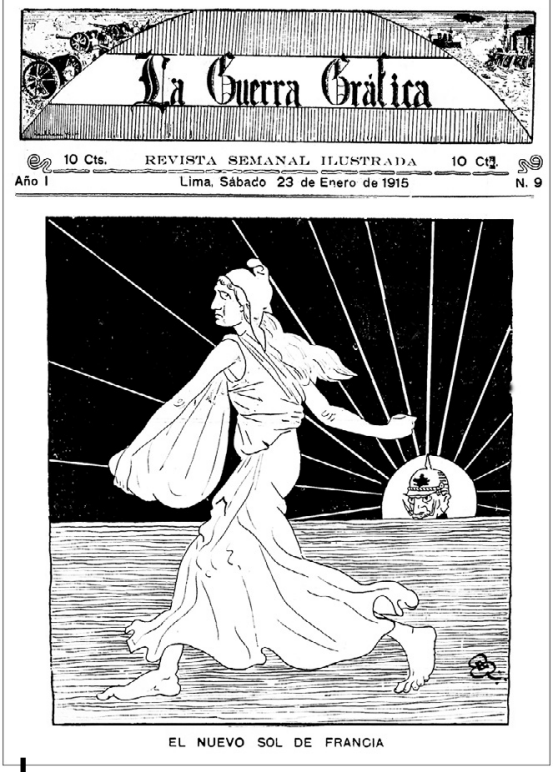

Figura 6 - «El nuevo sol de Francia»

La Guerra Gráfica, 23 de enero de 1915 
Ahora bien, si Francia está ridiculizada en la publicación, el discurso sobre el enemigo francés no deja de ser respetuoso. En su séptimo número, el periódico sacó en primera plana un largo artículo redactado por Maximiliano Harden, «célebre redactor del periódico 'Die Zukunft'», en el cual el autor invitaba a los ciudadanos franceses al diálogo. El discurso es bélico, pero atestigua cierto respeto por el enemigo:

Ya no tenemos tiempo para frivolidades. Tenemos que descubrirnos francamente y sin rodeos, las miras que llevamos. Se trata nada menos que de la existencia de vuestro país, de vuestro espíritu nacional; y nosotros nos preguntamos si debemos destruir lo que anhelamos conservar; porque le tenemos cariño a vuestra patria, así como a sus campeones inteligentes, aunque irreflexivos. Mañana será tarde. Os engañan, os estáis engañando. Vuestro ejército se ha batido con bravura. Hoy mismo se muestra valeroso [...]. Alemania está orgullosa de semejante enemigo, pero también firmemente convencida de que lo volverá a humillar [...] (La Guerra Gráfica, 9 de enero de 1915: 1)

A cambio, el discurso sobre Inglaterra era mucho más agresivo. En efecto, se observa respecto a la potencia británica, una inversión del discurso tradicionalmente asociado a la propaganda aliada destinada a desacreditar el imperio alemán. La Guerra Gráfica y Germania presentaron a los ingleses como crueles, conquistadores, inhumanos y bárbaros. Los ejemplos de este argumento son numerosos. En su número del 28 de abril de 1917, Germania recordó la violencia del ejército británico a lo largo de su historia. Mencionaba la guerra de los boers en Sudáfrica a finales del siglo XIX, y la crueldad de los soldados ingleses en la India (Germania, 28 de abril de 1917: 1), como una prueba de la barbarie intrínseca del enemigo británico. El 14 de julio del mismo año, Germania publicó un artículo en La Unión de Lima, en el cual se denunciaba el imperialismo inglés, ahí llamado «panbritanismo» (Germania, 14 de julio de 1917), neologismo derivado del término «pangermanismo». El argumento era muy claro: si bien al decir de la propaganda aliada, el imperio alemán tenía pretensiones territoriales expansionistas, Inglaterra no se quedaba atrás. La Guerra Gráfica también denunció la agresividad de la potencia británica en sus relaciones internacionales. El 2 de enero de 1915 publicó las líneas siguientes:

La hipocrecía [sic.] con que Inglaterra disfraza su asalto contra los alemanes con la violada neutralidad de Bélgica podría hacernos reír si los tiempos no fueran demasiado serios. Cada hoja de la historia comprueba que Inglaterra nunca se ha cuidado del derecho internacional, o solamente cuando las prescripciones de este derecho estaban identificadas con sus propios deseos [...](La Guerra Gráfica, 2 de enero de 1915: 12).

Este tipo de artículo tenía como claro objetivo desacreditar la beligerancia británica, al presentarla como ilegal, además que acompañada de una gran brutalidad. Esta última se vio ilustrada en La Guerra Gráfica por una serie de denuncias en contra de la artillería inglesa y del uso de las balas «Dum-Dum». Con lujo de detalles técnicos, la revista publicó varios artículos e ilustraciones sobre la crueldad del uso de este tipo de municiones.

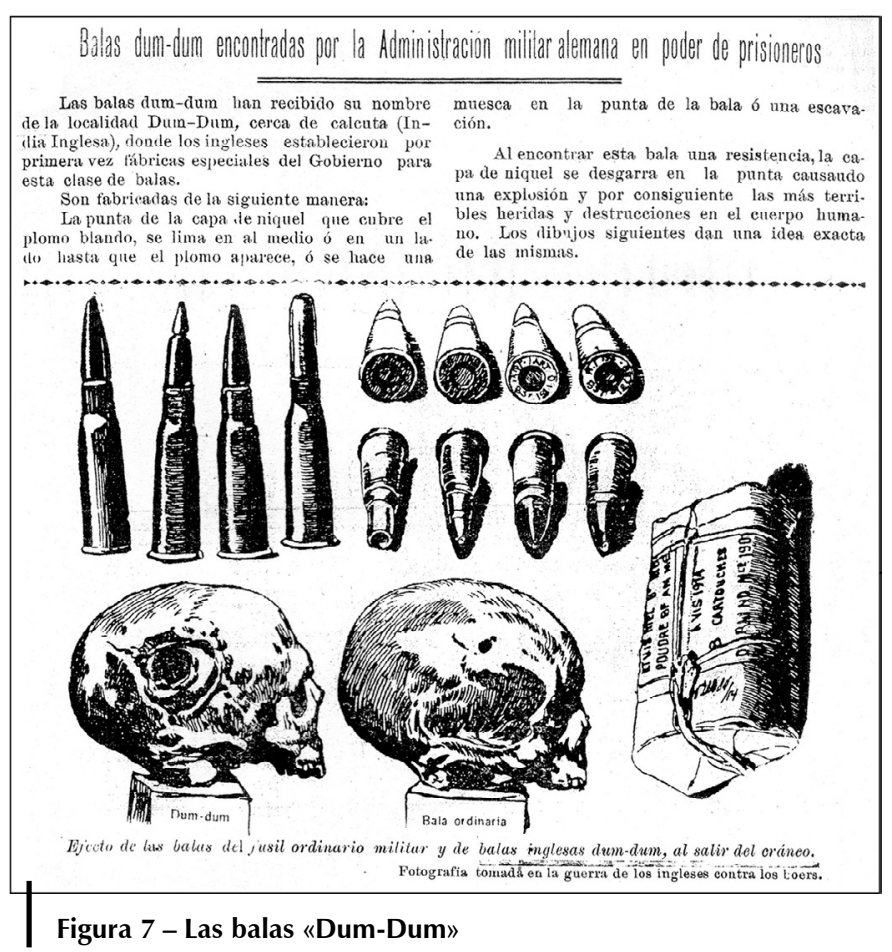

La Guerra Gráfica, 5 de diciembre de 1914: 9

Considerado como una violación de los acuerdos internacionales, el uso de este tipo de municiones fue denunciado a menudo por la publicación, y presentado como una prueba más de la crueldad inglesa. La Guerra Gráfica mostraba fotografías explícitas de las horribles heridas provocadas por el proyectil sobre los soldados alemanes (La Guerra Gráfica, 20 de marzo de 1915: 6), así como artículos denunciando la barbarie de tal práctica militar:

La bala dum-dum ha sido inventada para la seguridad de los hombres contra las bestias del Asia y África. Un tiro destroza al animal más grande. La convención de la Haya prohíbe terminantemente el uso de semejantes instrumentos de asesinato contra seres humanos. El empleo de balas dumdum enseña, en que bajo nivel se encuentra 'el pueblo de la cultura', los ingleses!! (La Guerra Gráfica, 23 de enero de 1915: 10).

En su empresa de denuncia sistemática de la barbarie y de la ilegitimidad de la beligerancia británica, La Guerra Gráfica recurrió a cualquier documento y anécdota susceptible de asentar su perspectiva, relegando a veces la precisión factual y la autenticidad de las fuentes en segundo lugar.

El 20 de enero de 1915, el periódico sacó a la luz una carta supuestamente redactada por una señorita inglesa y dirigida a su hermano, soldado en el frente; la carta posteriormente fue encontrada por un soldado alemán: 
Querido hermano: Estoy contenta de saber que estás en buenas condiciones. Quisiera estar contigo y quisiera ser hermana de caridad. Estoy segura que podría matar a uno o dos alemanes.

Estas líneas están acompañadas de este simple comentario, redactado por los editores de La Guerra Gráfica:

Dejamos los comentarios para el público, si las hermanas de caridad inglesas se dedican a matar los heridos que tienen a su cuidado (La Guerra Gráfica, 20 de febrero de 1915: 10).

Una vez más la crueldad británica parece ser demostrada, al caracterizar hasta las enfermeras inglesas. Numerosas son las historias así narradas por La Guerra Gráfica, sean sacadas del vivir cotidiano de los soldados en el campo de batalla o de la experiencia de los civiles en la retaguardia. Todas estas historias tienen un mismo objetivo y forman parte de un mismo aparato propagandístico, sencillo y claramente diseñado: revertir la dicotomía civilización/barbarie a favor del imperio alemán.

Ahora bien, y más allá de las orientaciones ideológicas del discurso, estas historias e ilustraciones del conflicto europeo contribuyen a la elaboración de un conjunto de representaciones sobre el conflicto, destinadas a un lectorado alejado de los campos de batalla.

\section{HACIA LA CONSTRUCCIÓN DE UNA «CULTURA DE LA GUERRA» LEJOS DE LOS CAMPOS DE BATALLA}

Definida a inicios de los años 1990 por especialistas franceses de la Gran Guerra (entre ellos los historiadores Stéphane Audoin-Rouzeau y Annette Becker), el concepto de «cultura de guerra» remite a la elaboración de un conjunto de representaciones sobre el conflicto por parte de las sociedades beligerantes. Objeto de apasionados debates entre los historiadores franceses, esta noción abrió la historiografía de la Primera Guerra Mundial al estudio cultural de un conflicto considerado como total. Si bien no entraremos en este debate historiográfico, consideraremos la elaboración de lo que Olivier Compagnon, a través de la revisión de la prensa argentina y brasileña, define como una «cultura de la guerra» (Compagnon, 2013a: 277-311).

Sobre este último punto, La Guerra Gráfica ofrece un espacio de exploración fructífero al brindar a su lector una amplia y diversificada propuesta de artículos e ilustraciones sobre el conflicto, a partir de los cuales se elabora una serie de representaciones sobre la guerra en sus diferentes dimensiones (sociales, políticas, humanas, etc.). La movilización de una gran diversidad de discursos y documentos en La Guerra Gráfica, ya fueran fotográficos, literarios o humorísticos, nos invita a considerar la construcción de cierta cultura bélica por parte de la comunidad alemana de Lima.

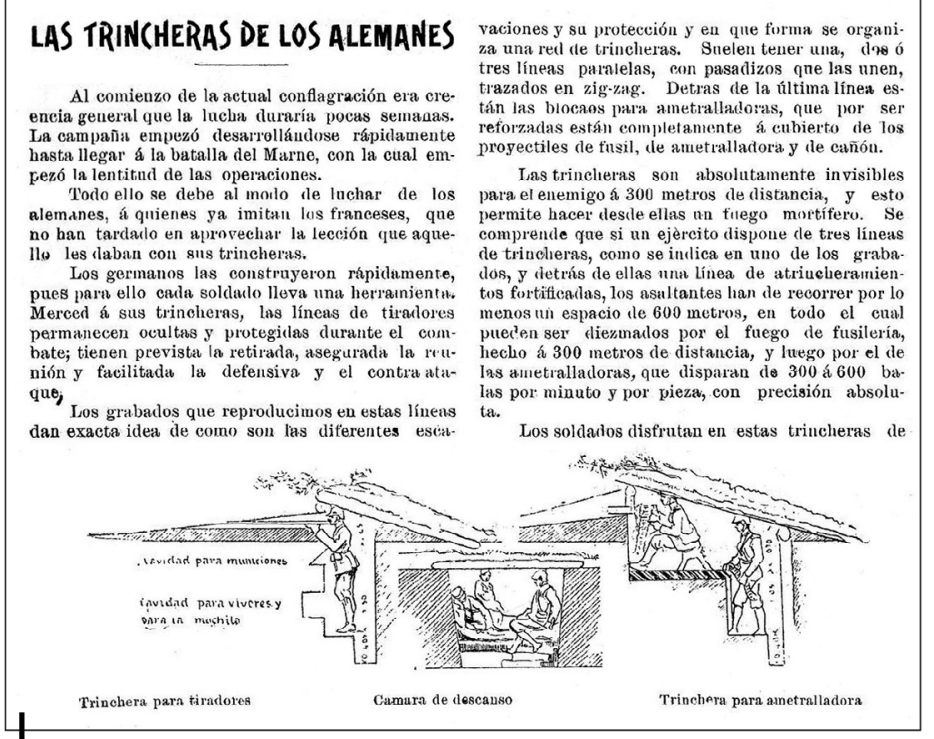

Figura 8 - Las trincheras alemanas

La Guerra Gráfica, 23 de enero de 1915: 1

En La Guerra Gráfica las representaciones del conflicto se elaboraron principalmente a través de un material gráfico diverso y de buena calidad: fotografías de soldados, del ejército y de su armamento, mapas, gráficos descriptivos de las trincheras, etc. Estas ilustraciones generalmente iban acompañadas por explicaciones diversas y datos técnicos. De manera general, la vida de los soldados en el frente de batalla fue objeto de numerosos artículos y fotografías, que ilustraban tanto la excelencia de la organización militar alemana, como la dificultad de la vida en las trincheras. Si bien la mayoría de las páginas de La Guerra Gráfica estuvieron dedicadas a esta descripción de la vida cotidiana de los soldados alemanes en el frente de batalla, la vida de los civiles en la retaguardia también fue objeto de numerosos apartados, articulados según una misma dicotomía: por un lado se trataba de valorar la eficacia del gobierno alemán, mientras que por el otro se subrayaba el sufrimiento de la población civil alemana. Entre diversos ejemplos, se puede mencionar este artículo publicado el 2 de enero de 1915 sobre las «Medidas para alimentar al pueblo alemán durante la guerra», en el cual se detallaban las leyes dictaminadas por el Reichstag para evitar la especulación sobre los alimentos y el aumento de los precios básicos, y eso con el fin de mantener al pueblo alemán «libre de la muerte por hambre, predicada por Inglaterra»16 (La Guerra Gráfica, 2 de enero de 1915).

${ }^{16}$ Recordemos que desde los primeros meses del conflicto, Inglaterra organizó un bloqueo marítimo del comercio alemán, precisamente con el objetivo de asfixiar la economía alemana y matar de hambre a la población del Imperio). 
Con este afán de compartir la realidad de la guerra con sus lectores peruanos, La Guerra Gráfica también alimentó un imaginario literario alrededor del conflicto. En su número del 6 de marzo de 1915, La Guerra Gráfica anunció la creación de una nueva sección en el periódico, presentada con este título algo paradójico «Lecturas amenas de la guerra», e inaugurada con este objetivo:

con el deseo de hacer nuestra revista lo más interesante posible, ofrecemos en adelante a nuestros lectores en esta acción, una serie de bosquejos,

novelitas, etc. cuyo tema tratará, siempre de la actual guerra (La Guerra Gráfica, 6 de marzo de 1915: 11).

A partir de esta fecha y en cada número, se publicaron fragmentos de novelas, historias de amor y de aventuras que presentaban el conflicto desde la ficción y la emoción.

De la misma manera y con el mismo objetivo, La Guerra Gráfica publicó también una sección «Humorísticos», que presentaba diversas caricaturas y anécdotas cómicas sobre el conflicto, destinadas a ridiculizar al enemigo, al mismo tiempo que ofrecían cierta mirada sobre la manera en que se vivía el conflicto en Alemania. Entre diversos ejemplos, podemos citar la siguiente broma, publicada el 26 de diciembre de 1915:

Algunos niños en Berlín, quieren jugar 'guerra' y pelean quienes deben representar a los serbios, a austriacos, a alemanes, y a los rusos. Todas las naciones están repartidas solamente Ruso no quiere ser nadie; en esta disputa grita uno de los muchachos señalando a otro: Luis U...tú has tenido una vez piojos, y además nunca te lavas, tú debes ser Ruso! (La Guerra Gráfica, 26 de diciembre de 1915: 14).

Para terminar, y esta vez en una tonalidad más trágica, La Guerra Gráfica se empeñó en transmitir a sus lectores la profunda emoción experimentada por la población alemana durante el conflicto, una población que presenciaba el sacrificio de su juventud en el frente de batalla. La revista publicó diversas cartas de soldados alemanes enviadas a sus familiares, en las cuales se describía la penosa vida en las trincheras. En estas cartas se mencionaban los problemas de higiene, de cansancio y la falta permanente de sueño, la mala alimentación, la lluvia que transformaba en lodo el piso de las trincheras, la violencia de los enfrentamientos con el enemigo, el miedo. También se mostraban diversas fotografías que sugerían al lector la soledad del soldado en el frente de batalla: la fotografía de un «marinero de un barco de guerra alemán leyendo una carta de sus parientes junto al Árbol de Noël [sic.], instalado a bordo para celebrar las Pascuas» o la de unos «soldados alemanes, convalecientes de sus heridas, celebrando la Navidad en la sala de heridos graves de un hospital berlinés» (La Guerra Gráfica, 27 de febrero de 1915: 10-12).

Gracias a estas ilustraciones y descripciones, el lector peruano podía tomar conciencia del horror de un conflicto que se estaba desatando a miles de kilómetros. El 30 de enero de 1915, el periódico publicó la fotografía de un grupo de infantes en los brazos de soldados, frente a las ruinas de lo que parecía haber sido un pueblo. La descripción de la fotografía no dice ni la nacionalidad de los protagonistas, ni el lugar donde fue tomada esta imagen, pero resume con poesía y emoción la monstruosidad universal y anónima de la guerra, en una visión que bien se podría calificar de premonitoria:

El cuadro de dolor y de espanto que ofrece la barbarie de la civilización en las naciones beligerantes, es más sombrío y angustioso cuando muestra la desventurada situación de los niños desamparados. La santa caridad les brinda brazos amigos y protección de corazones nobles. Bajo el burdo paño de las guerreras militares, los pechos heroicos han sentido hondos estremecimientos de compasión. Pero al rodar de los días, estos niños huérfanos serán hombres heridos por el más vivo de los dolores. De su triste infancia llevarán clavado el aguijón terrible de la pena. Evocarán la visión trágica de la guerra destruyendo edificios, quemando pueblos, rociando con sangre generosa escombros calcinados, sepultando para siempre en un abismo de ruina y de maldad el bendito amor de la madre mancillada y muerta. Y un gesto de repulsión y de odio contraerá perpetuamente sus labios, donde florecerá como una maldición la enconada canción de la venganza (La Guerra Gráfica, 30 de enero de 1915: 7).

\section{CONCLUSIÓN}

Como medios de difusión del punto de vista alemán sobre la Gran Guerra, en un espacio público peruano ampliamente aliadófilo, La Guerra Gráfica y Germania ofrecen al historiador una documentación sumamente valiosa, que nos invita a formular principalmente tres reflexiones.

La primera remite a la cuestión del acceso de la sociedad peruana a una información actualizada y fidedigna sobre el conflicto, así como a la participación de la comunidad alemana del Perú en su difusión. Si bien parece que La Guerra Gráfica ha sido fundada como una reacción local de la comunidad alemana de Lima para contrarrestar una opinión pública nacional aliadófila, no nos es posible descartar del todo una eventual intervención de los servicios de propaganda alemanes en esta iniciativa periodística. No sabemos con certeza cómo fueron organizadas la edición y la difusión de estas publicaciones, ni tampoco la amplitud de su lectorado. Sin embargo el análisis de las dos publicaciones, articulado al conocimiento del contenido de la propaganda aliada, nos permite documentar de manera precisa esta «guerra de la información» inédita que caracterizó el primer conflicto mundial.

La segunda reflexión que se desprende del presente estudio tiene que ver con la movilización de las comunidades europeas del Perú en el conflicto, al alimentar de manera concreta los estudios ahora numerosos sobre esta polarización de la opinión entre «aliadófilos» y «germanófilos» en América Latina. Esta reflexión permanece vigente después de la Primera Guerra mundial, para plantearse en términos relativamente similares durante la Segunda Guerra Mundial.

Para terminar, se debe subrayar que a través de la publicación de una gran diversidad de documentos fotográficos, cartográficos, literarios, epistolarios e 
informativos, La Guerra Gráfica contribuyó a la elaboración de una cultura de la guerra en América Latina, en espacios alejados del conflicto pero que, a través de una serie de representaciones, trataron de mantener cierta cercanía con la realidad vivida en Europa.

\section{Referencias citadas}

\section{Prensa consultada}

El Comercio, 1914-1918

La Guerra Gráfica, 1914-1915

Germania, 1916-1917

La Reforma, 1915

La Federación, 1915-1916

BASADRE, J., 2005 [1939] - Historia de la República del Perú, Vol. 13, Lima: El Comercio. BONILLA, H. \& RABANAL, A., 1979 - La Hacienda de San Nicolás (Supe) y la Primera Guerra Mundial. Revista Economía, II (3): 3-47.

BORRAS, G., en prensa - «Moi mon colon celle qu'je préfère... » Entre objetos musicales, imágenes y versos. Recepciones de la 'conflagración europea' en Lima. (19141919). In: América Latina y la Gran Guerra. Una historia conectada (O. Compagnon, C. Foulard \& G. Martin, eds.); México: CEMCA, El Colegio de México, UNAM.

COMPAGNON, O., 2013a - L'Adieu à l'Europe. L'Amérique latine et la Grande Guerre, 394 pp.; París: Fayard.

COMPAGNON, O, 2013b - Latin America. In: The Cambridge History of the First World War, vol. I "Global War" (J. Winter, ed.): 533-555; Cambridge: Cambridge University Press.

CUENCA, A., 2006 - La colonia británica de Montevideo y la Gran Guerra, 350 pp.; Montevideo: Torre del Vigia Editores.

FRANZINA, E., 2000 - La guerra lontana: il primo conflicto mondiale e gli italiani d'Argentina. Estudios migratorios latinoamericanos, 44: 57-83

GODBERSEN, G., 2002 - La inmigración alemana en el Perú, 2 vols.; Lima: Servicios Generales Rene S.A.C.

KATZ, F., 1981 - The secret war in Mexico: Europe, the United States and the Mexican Revolution, xii + 659 pp.; Chicago: Illinois, University of Chicago Press.

LUEBKE, F. C., 1987 - Germans in Brazil. A comparative history of cultural Conflict during World War $l$, xiii $+248 \mathrm{pp}$. Baton Rouge, Londres: Louisiana State University Press.

MADUEÑO, V., 1981 - La Primera Guerra mundial y el desarrollo industrial del Perú. Estudios Andinos, 9 (17-18): 41-53.

MARTIN, G., en prensa - The First World War in Peru: national reception and regional impacts. In: Toward a history of the First World War in Latin America (O. Compagnon \& M. I. Tato, eds.); Francfort-Madrid: Vervuert, Iberoamericana.

MILLIES, D., 2007 - Echando raíces. 180 años de presencia alemana en el Perú, 65 pp. Lima: La Molina, Galería ICPNA (Instituto Cultural Peruano Norteamericano).

MORALES ERROCH, E., 2006 - El Perú y la Primera Guerra Mundial: el hundimiento de la Lorton. Derroteros de la Mar del Sur, 14: 107-124.
NOVAK TALAVERA, F., 2004 - Las relaciones entre el Perú y Alemania (1828-2003), 269 pp.; Lima: Pontificia Universidad Católica del Perú, Instituto de Estudios Internacionales (IDEI).

NOVAK TALAVERA, F., 2005 - Las relaciones entre el Perú y Francia (1827-2004), 321 pp. Lima: Pontificia Universidad Católica del Perú, Instituto de Estudios Internacionales (IDEI).

OTERO, H., 2009 - La Guerra en la sangre. Los Franco-argentinos ante la Primera Guerra mundial, 222 pp.; Buenos Aires: Editorial Sudamericana.

PERALTA RIVERA, G., 2011 - Antenor Orrego y la Bohemia de Trujillo (1914-1916), 444 pp.; Lima: Fondo Editorial del Congreso del Perú, Universidad Privada Antenor Orrego.

RIVIALE, P., 2008 - Una historia de la presencia francesa en el Perú, del Siglo de las Luces a los años locos, 244 pp.; Lima: Institut Français d'Études Andines, Instituto de Estudios Peruanos, Fondo Editorial del Congreso del Perú, Embajada de Francia en el Perú.

SCHULZE SCHNEIDER, I., 1993 - La propaganda alemana en México durante la Primera Guerra Mundial. Anuario del Departamento de historia, 5: 261-272; Madrid: Universidad Complutense.

SCHULZE SCHNEIDER, I., 1995 - Alemania y América. La llamada del Nuevo Mundo: 500 años de presencia alemana en América, 323 pp.; Madrid: Editorial MAPFRE.

TATO, M. I., 2011 - El llamado de la patria. Británicos e italianos residentes en la Argentina frente a la Primera Guerra Mundial. Estudios Migratorios Latinoamericanos, 71 273-292.

TATO, M. I., 2014 - Luring neutrals. Allied and German propaganda in Argentina during the First World War. In: World War I and Propaganda (Troy R. E. Paddock, ed.): 322-344; Leiden-Boston: Brill Academic Publishers. 